Pecvnia, Monográfico (2008), pp. 147-183

\title{
La percepción del entorno organizativo y la creatividad: Análisis de las dimensiones del clima laboral que determinan el comportamiento creativo del empleado en la empresa
}

\author{
María F. Muñoz Doyague \\ mfmund@unileon.es \\ Universidad de León \\ Organización de Empresas \\ Fac. de Ciencias Económicas y Empresariales \\ Campus de Vegazana, $\mathrm{s} / \mathrm{n}$ \\ 24071 León (España)
}

La creatividad se está convirtiendo en un fenómeno de especial interés para las organizaciones. Por ello, existe un interés creciente por comprender cuáles son los factores responsables del rendimiento creativo en los entornos laborales. A pesar de ello, todavía existen lagunas importantes en la literatura sobre como influye la existencia en la empresa de un clima que apoye a la innovación. Con el fin de aportar nueva evidencia empírica al respecto, el objetivo del presente trabajo consiste en analizar cómo influye en la creatividad de los empleados, la percepción que éstos tengan de que el clima existente en la organización apoya a la innovación. Para ello, utilizando el concepto de clima psicológico
Creativity is becoming a particularly important phenomenon for organizations. There is consequently increasing interest in understanding the factors responsible for creative performance in labor environments. In spite of this, important gaps still exist in the literature about the influence of the work environment on creativity. With the aim of offering new empirical evidence on this question, the object of the current work is to analyze how employees' perception about the organizational climate influences their creativity. With this aim, using the concept of psychological climate and adopting a definition 
y adoptando una definición de creatividad en términos de producto, se realizó un estudio entre los empleados de una empresa del sector de la automoción. Tras someter la medida del clima a un análisis factorial por el método de componentes principales, que reveló la existencia de cinco dimensiones significativas, se realizó un análisis de regresión para ver la influencia de cada factor en la creatividad de los trabajadores. Los resultados logrados revelan la importancia que tiene esta variable en la explicación del rendimiento creativo. De esta forma se ha puesto de manifiesto que la empresa debe crear un entorno laboral favorable a la creatividad, mediante los mecanismos adecuados a tal efecto. Así, la provisión de recursos para la generación y el desarrollo de ideas, la existencia de una comunicación fluida en el seno de la empresa, la participación de todos los trabajadores en la toma de decisiones o la consideración del esfuerzo realizado parecen ser de especial relevancia para conseguir este propósito.

Palabras clave: clima psicológico, creatividad. of creativity in terms of the product, the work studies the employees of a firm from the automotive sector. We carried out a principal components factor analysis, with the aim being to obtain the dimensions or factors summarizing the main policies or procedures followed by the firm in favor of creativity. We obtained five significant dimensions and then, made a regression analysis in order to determine how each of the related to creativity. The findings reveal the importance of this variable for explaining creative performance. Thus, providing resources for the generation and development of ideas, the existence of a fluid communication within the firm, the participation of all the workers in the decisionmaking, and the recognition of workers' efforts all seem to be particularly relevant for achieving this purpose.

Key words: psychological climate, creativity.

\section{INTRODUCCIÓN}

En las últimas décadas, en las que la innovación se ha convertido en un elemento indispensable para aquellas empresas que deseen ser competitivas, saber cómo estimular el potencial creativo de la fuerza de trabajo es, sin lugar a dudas, una cuestión de creciente interés para las organizaciones. De hecho, existe acuerdo sobre el hecho de que la creatividad es un aspecto clave para garantizar el éxito del proceso de innovación. Incluso se ha llegado a afirmar que la creatividad, antes que la innovación, es la verdadera fuente de ventaja competitiva para las organizaciones, al ser la base de su potencial innovador (Amabile 1983, 1988, 1996; Woodman et al. 1993; Amabile et al. 1996; Ford 1996; Cummings y Oldham 1997).

Al igual que otros resultados organizativos, la creatividad tiene su origen en los individuos quienes, solos o con otros compañeros, llevan a cabo el trabajo diario de la organización. Cuando los empleados trabajan creativamente, sugieren productos, ideas o procedimientos nuevos y aprovechables que proveen a la organización de materia prima para que la empresa innove (Amabile 1988; Staw 1990; Woodman et al. 1993; Ford 
1996). Es decir, están proporcionando opciones a la organización, puesto que los inputs creativos posibilitan que ésta elija entre un conjunto más amplio de ideas para su desarrollo y posterior implantación (Cummings y Oldham 1997). El comienzo y puesta en marcha de estos productos fomenta la capacidad innovadora de una firma al otorgarle la flexibilidad necesaria para dar respuesta a las demandas y oportunidades externas y, por tanto, para adaptarse, crecer y competir (March y Simon 1958; Kanter 1984, 1988; Van den Ven 1986).

En este sentido, la mayor parte de la literatura que analiza los factores determinantes de la creatividad individual en las organizaciones, se han centrado en los atributos que tienen que reunir los empleados para ser creativos, ya sea en los rasgos de su personalidad (ej. Guilford 1950, 1977, 1984; Rhodes 1987; Findlay y Lumdsen 1988; Cummings y Oldham 1997), ya sea en otros elementos que pueden estimularse mediante prácticas adecuadas de gestión, como la motivación intrínseca (ej. Amabile 1988; Amabile et al. 1994; Ford 1999; Tierney et al. 1999; Taggar 2002), la experiencia (ej. Amabile 1988; Woodman et al. 1993; Ford 1996; Weisberg 1999; Taggar 2002; Tierney y Farmer 2002) o el estilo cognitivo (Ettlie y O'Keefe 1982; Payne et al. 1990; Baer 1994; Scott y Bruce 1994; Cummings y Oldham 1997; Tierney et al. 1999; Taggar 2002). Sin embargo, hasta que punto los trabajadores producen ideas creativas depende no sólo de sus características individuales, sino también del entorno de trabajo que perciban que les rodea (Amabile et al. 1996).

En este sentido, Angle (2000) señala que el comportamiento es una función de la persona y el entorno, lo que implica dos aspectos relevantes para la creatividad y la innovación organizativas: (i) las características personales y el contexto desempeñan roles por igual en ocasionar el comportamiento innovador y (ii) la interacción entre la gente y el contexto tendrá como consecuencia resultados que no son conseguidos de ningún modo si se consideran ambos aspectos por separado. Por tanto, el contexto puede ser un arma de doble filo, de forma que en ocasiones restringe el comportamiento innovador o, por el contrario, lo hace más probable.

Se sabe además, que el entorno laboral tiene consecuencias en la efectividad y en la eficacia organizativas (Katz y Kahn 1978) y es una parte ineludible de la experiencia diaria de todo trabajador (Turnipseed 1994), por lo que es razonable esperar que tiene el potencial de ejercer una influencia considerable en el comportamiento humano (Pritchard y 
Karasik 1973) y, por extensión, en todos los aspectos del desempeño organizativo, incluyendo la creatividad.

En esta misma línea, Galbraith (1982) señala que si puede estructurarse un entorno para estimular la creatividad, los comportamientos creativos resultantes pueden contribuir a la productividad y al grado de innovación a largo plazo de la organización. Según Mumford y Gustafson (1988), incluso cuando los individuos han desarrollado la capacidad para ser creativos, su voluntad para emprender esfuerzos productivos esta dirección puede estar condicionada por las creencias relativas a las consecuencias de tales acciones en un entono dado. Ello entraña, por tanto, que la presencia de gente creativa no garantiza per se que la organización sea creativa e innovadora, sino que es necesario algo más. Ese algo más es el ambiente de trabajo que rodea a los individuos, por lo que importante identificar cuáles son los elementos del entorno laboral que facilitan el esfuerzo creativo.

Amabile (1996: 17) refuerza esta última conjetura, al afirmar que "cualquiera que sean los talentos de un individuo... las condiciones bajo las cuáles trabaja... pueden incrementar o disminuir significativamente el nivel de creatividad producida". Esta aseveración está presente en todo el trabajo de esta autora, en el que subyace la premisa de que todos los seres humanos con capacidades normales son capaces de producir alguna vez trabajo creativo en algún área o campo de trabajo, al menos moderadamente, y que el contexto social influye significativamente tanto en el nivel como en la frecuencia de comportamiento creativo del individuo.

Oldham y Cummings (1996) apoyan esta idea, al demostrar que la interacción entre la persona y la situación tiene un efecto en la creatividad de los individuos, de forma que aquellos individuos cuyas disposiciones les hacen más susceptibles de ser afectados por un entorno laboral favorable, son más creativos, pero que la creatividad de todos los individuos aumenta cuando están inmersos en un entorno que les apoya en este sentido.

Así, frente a las perspectivas tradicionales que entienden la creatividad como una entidad todo o nada, hoy en día, se asume que todos los individuos con aptitudes normales son capaces de producir trabajo creativo, al menos moderadamente y que, además, el ambiente social puede influir tanto en el nivel como en la frecuencia del comportamiento creativo (West y Farr 1990; Amabile 1997). Esta conclusión pone de 
manifiesto la importancia de articular un entorno laboral que estimule la creatividad puesto que, con independencia de los talentos o características de una persona, las condiciones bajo las cuáles trabaja pueden aumentar o disminuir de forma significativa su nivel de creatividad.

Desde esta perspectiva, el clima ha sido considerado como un importante factor del contexto que influye en la creatividad individual (Cummings 1965; Mumford y Gustafson 1988; Woodman et al. 1993; Tesluk et al. 1997). Es claro que cuando hablamos de la percepción que tiene un empleado del entorno en el que trabaja, aglutinamos el conjunto de características que definen el entorno de trabajo. En este sentido, Zhou y George (2001) apuntan que las organizaciones deben elegir enfatizar su apoyo a la creatividad, en virtud del grado en el cuál sus empleados lo perciban así, es decir, en tanto en cuanto éstos aprecien que la organización estimula, respeta, recompensa y reconoce a quienes generan ideas novedosas y útiles. Bajo estas circunstancias, en las que los empleados sienten que la creatividad es apoyada y valorada por su organización, el riesgo potencial asociado con el hecho de ser creativo se minimiza y aumenta la sensación de que las ideas creativas son eficaces, lo que redundará en una mayor creatividad individual.

Por tanto, el objetivo de este trabajo consiste en analizar cómo influye el clima organizativo percibido por los empleados en su producción creativa, por considerar que aúna los diversos aspectos que pueden contemplarse dentro del contexto organizativo, al ser un constructo multidimensional y que, generalmente, es concebido como una variable esencial para comprender el comportamiento de las organizaciones y sus miembros (Dastmalchian 1986). La causa de esta última afirmación está en que el clima afecta enormemente a la habilidad de la organización para utilizar sus recursos técnicos y humanos (Isaksen y Kaufmann 1990). Así, "la capacidad técnica,... es esencial para el éxito de la organización, pero el que esa capacidad sea o no canalizada hacia actividades productivas, depende del clima" (Friedlander y Margulies 1969: 173) ${ }^{1}$. 


\section{LA CREATIVIDAD}

La creatividad es un constructo complejo y difuso que ha sido definido de diversas maneras (Mumford y Gustafson 1988; Shalley 1995). Así, se ha propuesto que la creatividad puede ser identificada con las características concretas de productos (ej. Zaltman et al. 1973; Amabile 1982; Shalley 1991; Woodman et al. 1993; Oldham y Cummings 1996), de personas (ej. Guilford 1950; McKinnon 1962, 1965; Torrance 1974), de los procesos de pensamiento (ej. Wallas 1926; Wertheimer 1945; Koestler 1964; Stein 1974; Weisberg 1986, 1993) o de la situación en la que tiene lugar (Csikszentmihalyi 1988, 1990; Gruber 1988).

No obstante, a pesar de que ello ha supuesto la carencia de integración en la investigación sobre la materia (Mumford y Gustafson 1988), existe acuerdo en literatura sobre el hecho de que la creatividad hace referencia a algo que es novedoso y en algún sentido valioso (Ford 1995). El establecimiento de una estrategia o solución creativa variará según el campo de actuación o según la tarea implicada, pero todos los comportamiento creativos implican en alguna medida la identificación de formas originales y mejores de lograr algún propósito (Shalley 1995).

Por otro lado, la mayoría de los investigadores en la materia que se han centrado en el estudio de la creatividad en las organizaciones, han adoptado una definición que se centra en el producto o idea generada, puesto que una persona, proceso o situación son creativos si han generado un producto que pueda ser calificado como tal (McKinnon 1978; Rhodes 1987). En este sentido, la definición más aceptada es la que apunta que la creatividad implica la producción o el desarrollo de ideas, procesos o procedimientos nuevos y útiles por un individuo o grupo de individuos que trabajan juntos (Amabile 1983, 1988; Shalley 1991; Woodman et al. 1993).

De acuerdo con esto, en este trabajo se considera que la creatividad es la generación por parte de un individuo de ideas novedosas y útiles para la organización. Una idea o producto será novedoso si implica, bien la recombinación significativa de los materiales existente, bien la introducción de materiales completamente nuevos (Oldham y Cummings 1996); además esas contribuciones deben ofrecer algo original y único en relación a lo que ya está disponible en el repertorio de productos o procedimiento de la empresa (Cummings y Oldham 1997). Una idea o producto será útil cuando sirven para solucionar un problema, ajustarse a las exigencias de una situación dada o lograr alguna meta 
reconocible (McKinnon 1978), lo que implica que debe ser directamente relevante para las metas de la organización y ser algo de lo cual ésta pueda esperar extraer algún valor, ya sea en el corto o en el largo plazo (Cummings y Oldham 1997).

\section{EL CLIMA Y LA CREATIVIDAD}

Desde una perspectiva subjetiva, el clima organizativo es considerado como la estructuración cognitiva y perceptiva de la situación organizativa común a todos sus miembros (Ekvall 1987). De forma más precisa el clima son "las percepciones compartidas sobre las políticas, prácticas y procedimientos organizativos, tanto formales como informales" (Reichers y Schneider 1990: 22). En concreto, consta de un conjunto de características que son percibidas por los miembros de la organización y que (i) describen la empresa, (ii) la distinguen de otras, (iii) es relativamente duradero en el transcurso del tiempo y (iv) tiene influencia sobre las personas dentro de ella y se dirige a ellas (Forehand y Gilmer 1964); es decir, para los miembros de una organización, el clima se refleja en las actitudes y expectativas que mantienen hacia la organización (Siegel y Kaemmerer 1978). Así, el clima de trabajo es una cualidad relativamente duradera del entorno interno de una organización que la diferencia de otras organizaciones y que es el resultado del comportamiento y las políticas de los miembros de la organización, especialmente la alta dirección, es percibido por éstos, sirve como base para interpretar la situación organizativa y actúa como una fuente de presión para dirigir la actividad (Pritchard y Karasick 1973; Kopelman et al. 1990).

De acuerdo con esta perspectiva, en la organización se da un flujo continuo de eventos y acciones, de rutinas y procesos, de forma que cuando los individuos afrontan tales fenómenos, intentan interpretarlos para hacer comprensible el entorno que les rodea, creando su propio "mapa cognitivo". Según Ekvall (1987), cuando los miembros de una organización interactúan, se produce un intercambio de experiencias e introspecciones, de forma que sus mapas cognitivos personales se confrontan entre sí y se modifican, surgiendo así las formas comunes de percibir e interpretar lo que sucede en la organización. Por tanto, el clima se centra en las percepciones de los miembros de una organización acerca de cómo son las cosas, pero dicha percepción incluye la idea de que tal significado está vinculado al evento o cosa percibido (Bruner 1964). 
Según Kopelman et al. (1990), los entornos laborales pueden ser representados de forma característica como:

un manojo de estímulos que presentan indicios ambiguos y conflictivos. Así mismo, los miembros de una organización deberían ser vistos como receptores e intérpretes activos de sus entornos laborales. Esos entornos así percibidos y procesados pueden ser concebidos como descripciones psicológicamente significativas de contingencias e influencias situacionales que los individuos utilizan para aprehender, disponer, predecir resultados y calibrar la corrección de sus comportamientos organizativos (pp. 294-295).

Esta descripción del entorno de trabajo basada en la cognición del ser humano ha sido utilizada por diversos autores como Campbell et al. (1970), James y Jones (1974), o Schneider (1975) y ha sido denominada clima psicológico, término acuñado por James y Jones (1974) y Jones y James (1979).

Estos últimos, a partir de una revisión sobre la teoría y la investigación del clima organizativo, proponen una clasificación conceptual que ha tenido una gran aceptación en la literatura académica. Así, distinguen entre "clima organizativo", cuando éste es considerado un atributo de la organización, y "clima psicológico" cuando el clima es considerado como una característica individual. De acuerdo con Ekvall (1987), James y Sells (1981) y James et al. (1990), el clima psicológico debería utilizarse en aquellos estudios en los cuales se utilicen las descripciones de los individuos como unidades de análisis, sin ningún intento de agregación para generar una medida organizativa, por lo que será la perspectiva que se utilizará en esta investigación. En este sentido, James y Jones (1974) identificaron el término clima psicológico como consistente con el enfoque de medición que pide a los individuos que den cuenta de sus percepciones sobre el entorno de trabajo y como el término preferido cuando esas percepciones individuales son utilizadas como la unidad de análisis. Puede decirse que el clima organizativo entraría dentro del enfoque objetivo mientras que el psicológico se acerca a la perspectiva subjetiva.

Por tanto, se entiende por clima psicológico, la percepción y descripción que hacen los individuos de la situación organizativa, como participantes en la vida de dicha organización (James y Jones 1974; Payne y Pugh 1976; Joyce y Slocum 1982), es decir, las percepciones que valoran la importancia y el significado de los entornos de trabajo para los 
individuos (James y James 1989). Dicho de otro modo, el clima psicológico representa valoraciones de acontecimientos, objetos, procesos y estructuras ambientales, lo que es decir evaluaciones cognitivas de los atributos ambientales en términos del significado e importancia que han adquirido para el individuo (James et al. 1990), es decir,

son representaciones cognitivas de los individuos acerca de eventos situacionales relativamente próximos que reflejan el significado y la importancia psicológica de la situación para el individuo. Una premisa central de la teoría del clima psicológico es que los individuos tienden a interpretar situaciones en términos psicológicos; es decir, a asignar un significado psicológico a los atributos y eventos ambientales... el clima psicológico es considerado como un atributo del individuo (James y Sells 1981: 275).

El clima para la creatividad tiene que ver con el grado en el cuál los individuos perciben que las políticas, prácticas y procedimientos organizativos especifican metas relacionadas con el desarrollo de nuevos productos, ideas, servicios o procesos, al tiempo que proporcionan los medios para funcionar creativamente (Tesluk et al. 1997). Diversos trabajos teóricos han sugerido que el clima puede canalizar y dirigir la atención y las actividades de la organización hacia la creatividad y la innovación (ej. Isaksen 1987; Amabile 1988; Woodman et al. 1993; Ford 1996).

De acuerdo con Isaksen (1987) y Taylor (1972), el análisis del clima conducente a la creatividad es un aspecto esencial en las investigaciones sobre este fenómeno. Isaksen y Kaufmann (1990) consideran que concurren diversos beneficios derivados de una comprensión del clima para la creatividad existente en la organización. En concreto, a nivel individual, tal entendimiento (i) ayuda al individuo a apreciar más claramente las percepciones del entorno, haciendo lo invisible un poco más visible, (ii) puede contribuir a que éste identifique y llegue a estar más implicado en moldear el clima en el que trabaja y (iii) como resultado de este mejor conocimiento, los individuos pueden ser capaces de reducir ciertos aspectos negativos y centrarse en los aspectos más positivos de sus percepciones del clima de trabajo. A nivel organizativo los beneficios pasan por (i) ayudar a determinar la idoneidad del clima para el éxito de la organización, es decir, si se ajusta a los propósitos de la empresa, (ii) permitir determinar el ajuste del clima a las necesidades de los recursos humanos y (iii) poder permitir que la organización se estructure mejor de cara a promover la creatividad y cubrir mejor sus necesidades. 
Los estudios del clima organizativo y la productividad llevados a cabo por Taylor $(1963,1972)$ y Andrews (1975) indican que un clima organizativo que proporcione apoyo físico a los esfuerzos creativos y estimule la acción independiente, tiende a facilitar la productividad científica. Pelz (1956) demostró que los climas que estimulan la interacción, la autonomía individual y la producción de conocimiento generaban logros creativos, mientras que los que se caracterizaban por la desconfianza, la carencia de comunicación, la ambigüedad de las metas y por limitar la autonomía individual, inhibían la innovación científica.

Igualmente, los estudios de Ellison et al. (1968) y Ellison et al. (1969) muestran que las percepciones individuales sobre el apoyo, la confianza, la comunicación y la claridad de las metas están correlacionadas positiva y significativamente con el logro científico.

Por tanto, la literatura académica sugiere que el clima que facilita la innovación es aquel que proporciona una base cognitiva para la generación de la idea y estimula las acciones requeridas para implementar esas ideas, al tiempo que demuestra aceptación y reconocimiento por los esfuerzos creativos del individuo (Mumford y Gustafson 1988). No es sorprendente entonces que, en su estudio sobre el clima de trabajo en organizaciones de I+D, Abbey y Dickson (1983) encontraran que éste se caracteriza por el reconocimiento y la recompensa del rendimiento superior, concretamente en las fases exploratorias iniciales de esfuerzos creativos, en las cuáles las necesidades individuales de aceptación y apoyo juegan un papel importante en la motivación para la realización de esfuerzos ulteriores.

Según Mumford y Gustafson (1988) la creatividad y la innovación parecen surgir en entornos capaces de proporcionar a los creadores recompensas personales significativas por los esfuerzos realizados, al tiempo que aseguran que el individuo posee las herramientas requeridas para lograr dichas recompensas. El logro creativo puede ser igualmente facilitado por un entorno que proporcione una base cognitiva por los esfuerzos creativos mediante estructuras que estimulen la exploración continua de puntos de vista alternativos. Por último, un entorno que proporcione un marco para la acción consistente con las características esenciales asociadas con los cometidos creativos, tales como la independencia o la auto confianza, parece probable que contribuya al logro innovador. Por tanto, los sistemas organizativos que apoyen la autonomía o generen la autoestima pueden incrementar la probabilidad del rendimiento creativo. 
Torrance $(1962,1967)$ sugiere que en un clima creativo debe haber respeto por las cuestiones e ideas inusuales y oportunidades para que el desempeño tenga lugar sin elevados niveles de aprensión por la evaluación.

Amabile y Conti $(1997,1999)$ también analizaron los cambios que sufre el entorno de trabajo creativo antes, durante y después de un proceso importante de downsizing en una gran organización de alta tecnología. Los resultados del estudio revelaron que los aspectos percibidos del entorno que más apoyan la creatividad disminuyen significativamente durante el downsizing y que, posteriormente vuelven a incrementarse aunque de forma modesta. Este hecho pone de manifiesto nuevamente la importancia de la gestión del entorno como forma de evitar o aliviar los efectos negativos en la creatividad de un proceso de estas características.

En un intento por comprender el clima para la creatividad, Amabile (1988) y Amabile y Gryskiewicz (1989), identificaron una serie de cualidades del entorno que bien estimulaban, bien inhibían la productividad creativa en diversos entornos organizativos. Así, a partir de una serie de entrevistas realizadas a científicos de I+D de más de 20 empresas (Amabile y Gryskiewicz 1989), y a empleados de marketing y desarrollo de un gran banco y al personal de ventas de una compañía de ferrocarril Amabile (1988), llegan a la conclusión de que la autonomía, una buena gestión del proyecto, el acceso a recursos suficientes, el estímulo, el reconocimiento mediante una retroalimentación positiva o las recompensas adecuadas, la concesión de tiempo suficiente para pensar en los problemas, el desafío y la presión por conseguir algo importante, son cualidades de los entornos organizativos que estimulan la creatividad de sus miembros. Entendiendo por éstas cualquier factor ajeno a los individuos en sí mismos, incluidas otras personas, que parecen influir de forma consistente en la creatividad, ya sea positiva o negativamente.

Posteriormente, Amabile et al. (1996) realizaron un estudio para determinar si existía una evidencia clara de la relación entre el entorno laboral percibido y la creatividad, cuestión fundamental que surge del modelo teórico. En su investigación examinaban el contexto psicológico para la creatividad, es decir, las percepciones del entorno de trabajo que pueden influir en el trabajo creativo llevado a cabo en las organizaciones. Para ello, realizaron un estudio de tres fases en una gran empresa de electrónica y alta tecnología que contaba con más 30.000 empleados. Las conclusiones obtenidas revelaron que los individuos que perciben que su organización, sus supervisores y sus grupos de trabajo 
estimulan y apoyan la creatividad, que su trabajo es desafiante, que tienen autonomía en la realización de sus tareas, amén de recursos suficientes y adecuados, son más creativos que aquellos que estiman que tienen una excesiva presión en el trabajo o que su organización se caracteriza por la existencia de problemas políticos internos, la crítica acerba de las ideas nuevas, la competición interna destructiva, la evitación del riesgo y un gran énfasis en el statu quo.

Estos resultados son, por otra parte, consistentes con los que recoge la literatura, tanto teórica como empírica, al respecto. Así, por ejemplo, el estímulo de la organización recoge diversos aspectos que encuentran apoyo, como es el caso del estímulo del riesgo para generar nuevas ideas junto con la valoración de la innovación a lo largo y ancho de la organización (Cummings 1965; Siegel y Kaemmerer 1978; Kimberly y Evanisko 1981; Kanter 1983, 1988; Delbecq y Mills 1985), la evaluación de apoyo y justa de las nuevas ideas (Cummings 1965; Kanter 1983; Shalley 1995; Shalley y Perry-Smith 2001), la expectativa de evaluación informadora frente a evaluación controladora (Rogers 1954; Amabile et al. 1990; Shalley 1995; Shalley y Perry-Smith 2001), que por otro lado se ha demostrado que también influye en la motivación intrínseca (Deci y Ryan 1985; Shalley et al. 1987), y la recompensa y reconocimiento de la creatividad (Cummings 1965; Paolillo y Brown 1978; Abbey y Dickson 1986), o la exposición a las ideas potencialmente relevantes de otros compañeros con los que se trabaja (Shalley 1995; Shalley y Perry-Smith 2001).

Por otro lado, diversos estudios han señalado la importancia de la función de los supervisores o jefes de proyecto en la creatividad de sus subordinados, más en concreto la claridad de las metas (Bailyn 1985; Shalley 1995), las interacciones abiertas entre supervisor y subordinado (Kimberly 1981; Kimberly y Evanisko 1981; Kanter 1988; Scott y Bruce 1994; Oldham y Cummings 1996; Tierney et al. 1999), o el apoyo del superior a las ideas del individuo o el grupo (Scott y Bruce 1994; Oldham y Cummings 1996; Tierney et al. 1999).

Igualmente, se ha demostrado que la creatividad aumenta cuando los individuos tienen una autonomía relativamente elevada en la realización y estructuración de sus tareas (Cummings 1965; Pelz y Andrews 1966; Andrews y Farris 1972; Cummings et al. 1975; Paolillo y Brown 1978; Bailyn 1985; Shalley et al. 2000), cuando los individuos perciben que tienen suficientes recursos (Taylor 1963; Kanter 1983; Delbecq y Mills 1985; Cohen y Levinthal 1990; Damanpour 1991) o cuando el trabajo supone un desafío (Andrews y Farris 1972; Amabile 1988; Amabile y 
Gryskiewicz 1989; Oldham y Cummings 1996) e impone una cierta presión en el tiempo de realización (Andrews y Farris 1972; Conti et al. 1996; Amabile et al. 2002).

Ello es debido a que las percepciones que tienen los individuos de estos aspectos provocan en ellos sentimientos positivos de que pueden elegir cómo realizar sus trabajos sintiendo que son responsabilidad suya, sobre el valor intrínseco que tienen para la organización los proyectos que han emprendido o de que su trabajo es importante y determinante para los objetivos de la organización respectivamente (Amabile et al. 1996) $)^{2}$.

En el lado de los aspectos negativos que influyen en la creatividad, también la literatura apunta hacia el conservadurismo, la rigidez y las estructuras de gestión formales (Kimberly 1981; Kimberly y Evanisko 1981), factores que, en esencia, son percibidos como controladores y que, por otro lado pueden también llevar a que descienda su motivación intrínseca, necesaria para la creatividad (Deci y Ryan 1985).

Por tanto, la caracterización por parte de la organización de todos estos aspectos incluidos en el entorno laboral determinará la percepción del clima psicológico que tengan los individuos, determinando su nivel de generación de ideas, productos, procesos, etc., nuevos y originales, es decir, su nivel de creatividad.

Al respecto, Tesluk et al. (1997), adaptan las dimensiones generales del clima de Kopelman et al. (1990), para describir el clima para la creatividad, relacionando cada una de ellas con las estructuras, prácticas, políticas y procedimientos organizativos, como se plasma en la Tabla 3.1, en la que se observa un gran paralelismo con la evidencia que ofrece la literatura académica.

Por otro lado, si bien el análisis de aspectos puntuales del entorno y su relación con la creatividad es, como se ha comentado, relativamente abundante, no lo es tanto la realización de estudios que investiguen la relación entre la variable clima organizativo percibido y la creatividad puesta de manifiesto por los empleados. No obstante, las

2 En estas ideas están implícitas las nociones de seguridad psicológica y libertad psicológica percibidas por el individuo, que vienen determinadas por el establecimiento de las condiciones ambientales adecuadas y que maximizan la probabilidad de que el individuo sea creativo (Rogers 1954). 


\section{conclusiones de todos ellos coinciden en señalar que la percepción por parte de los individuos de un clima de apoyo a la creatividad y la innovación es decisiva en el nivel de creatividad que muestren, tal y como se observa en la Tabla 3.2.}

Tabla 3.1: Ejemplos de prácticas, políticas y procedimientos y las dimensiones relacionadas del clima para la creatividad

\begin{tabular}{|c|c|}
\hline $\begin{array}{l}\text { Ejemplos de prácticas, políticas } \\
\text { y procedimientos de apoyo }\end{array}$ & $\begin{array}{l}\text { Dimensión correspondiente del } \\
\text { clima para la creatividad }\end{array}$ \\
\hline $\begin{array}{l}\text { Articulación clara de las metas para el desarrollo de } \\
\text { nuevos productos, servicios y procesos, por parte de la } \\
\text { alta dirección. } \\
\text { Comunicación de las metas a los empleados (ej. } \\
\text { declaración de la misión). }\end{array}$ & $\begin{array}{l}\text { Énfasis en las metas: grado en que las } \\
\text { metas para la creatividad y la innovación } \\
\text { y los estándares para lograrlas son dadas } \\
\text { a conocer a los empleados. }\end{array}$ \\
\hline $\begin{array}{l}\text { Prácticas directivas y de supervisión que estimulan } \\
\text { visionar los problemas desde múltiples perspectivas } \\
\text { (ej. uso de equipos multifuncionales). } \\
\text { Prácticas de implicación de los empleados que } \\
\text { promuevan la participación extensiva (ej. equipos } \\
\text { autogestionados, programas de sugerencias, equipos } \\
\text { de solución de problemas). }\end{array}$ & $\begin{array}{l}\text { Énfasis en los medios: grado en el que } \\
\text { los métodos y los procedimientos para la } \\
\text { creatividad y la innovación son transmi- } \\
\text { tidos a los empleados. }\end{array}$ \\
\hline $\begin{array}{l}\text { Prácticas de recompensas y reconocimiento que } \\
\text { estimulen la motivación intrínseca. } \\
\text { Evaluación de las nuevas ideas y sugerencias basada } \\
\text { en la contribución que aportan, no en el lugar que } \\
\text { ocupa el creador en la jerarquía organizativa. }\end{array}$ & $\begin{array}{l}\text { Orientación de las recompensas: grado en } \\
\text { que las recompensas y las evaluaciones } \\
\text { están asignadas sobre la base de los } \\
\text { resultados en creatividad e innovación. }\end{array}$ \\
\hline $\begin{array}{l}\text { Suministro adecuado de materiales, tiempo y dinero } \\
\text { para los esfuerzos en innovación. } \\
\text { Formación para desarrollar el conocimiento y las } \\
\text { habilidades criticos que apoyan los comportamientos } \\
\text { creativos. } \\
\text { Estructuras que proporcionen a los empleados apoyo } \\
\text { técnico y financiero para las nuevas ideas (ej. centros } \\
\text { de desarrollo de ideas). }\end{array}$ & $\begin{array}{l}\text { Apoyo a la tarea: grado en el cuál los } \\
\text { empleados creen que están siendo } \\
\text { apoyados pro asignaciones de tiempo, } \\
\text { dinero, equipamiento, materiales y servicios } \\
\text { necesarios para funcionar creativamente } \\
\text { e implantar las nuevas ideas, proyectos y } \\
\text { soluciones. }\end{array}$ \\
\hline $\begin{array}{l}\text { Estilos de dirección y supervisión que estimulen la } \\
\text { toma de riesgos y la colaboración activa, además de } \\
\text { proporcionar apoyo interpersonal. } \\
\text { Políticas de evaluación que no castiguen las acciones } \\
\text { bien intencionadas que terminan en fracaso, sino que } \\
\text { estimulan el aprendizaje por el error. }\end{array}$ & $\begin{array}{l}\text { Apoyo socioemocional: grado en el que } \\
\text { los empleados consideran que el entorno de } \\
\text { trabajo proporciona el apoyo interpersonal } \\
\text { necesario para sentirse libres para funcionar } \\
\text { creativamente. }\end{array}$ \\
\hline
\end{tabular}

Fuente: Tesluk et al. (1997). 
Tabla 3.2: Trabajos empíricos de la relación entre el clima y la creatividad

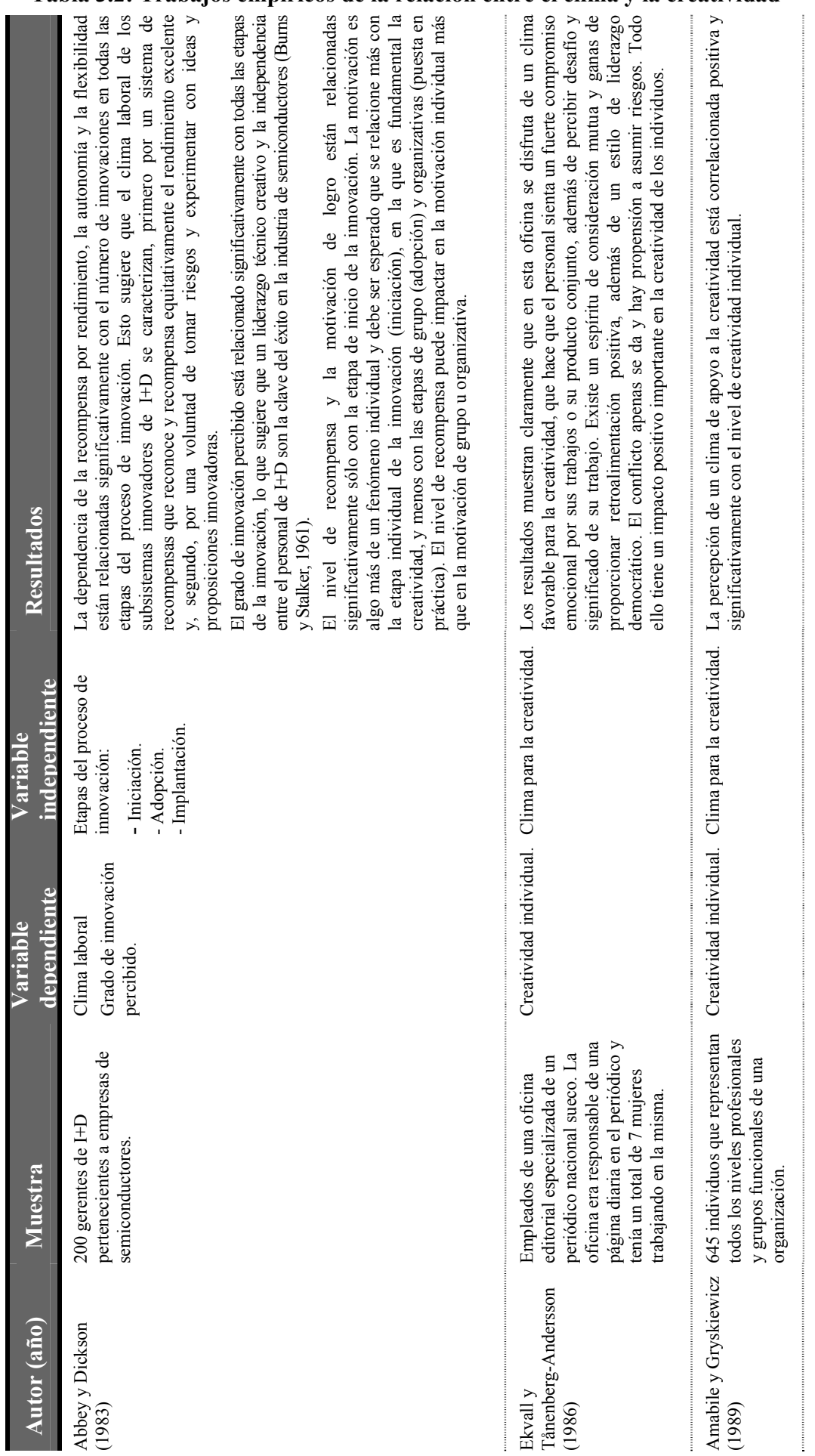


Tabla 3.2: Trabajos empíricos de la relación entre el clima y la creatividad (continuación)

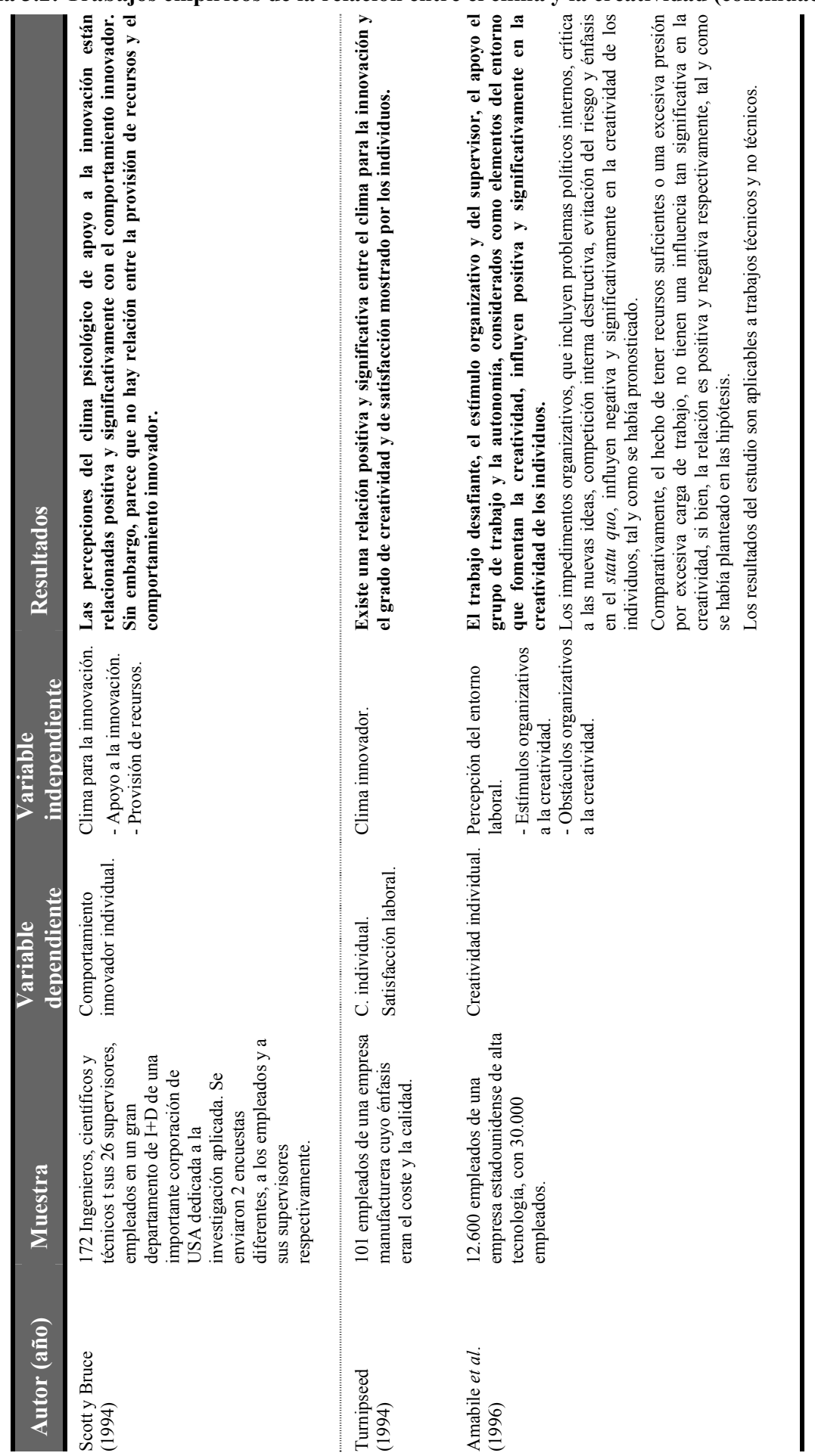


Así pues, dada una concepción del clima como un determinante del comportamiento individual y en base al apoyo empírico previo de los efectos del clima en la creatividad individual, se plantea la siguiente hipótesis:

H1: La percepción por parte de un individuo de un clima de apoyo a la creatividad influye positivamente en su rendimiento creativo.

\section{METODOLOGÍA}

\subsection{Muestra}

Para contrastar la hipótesis propuesta, se realizó el estudio entre los empleados de una empresa del sector de la automoción, cuya actividad principal consiste en la fabricación de vehículos de dos ruedas. Para ello, la entidad aplica la filosofía de producción justo a tiempo, en la que es fundamental la generación de ideas creativas para conseguir la mejora continua en la búsqueda de la calidad total.

La población objeto de estudio estaba formada por 110 trabajadores pertenecientes a las divisiones de administración, producción y ventas, quedando excluidos los miembros del ápice estratégico y los trabajadores de la cadena de montaje. A todos ellos se les envío un cuestionario en el que se les planteaba una serie de preguntas que tenían como finalidad medir las variables del modelo a contrastar. Se realizaron dos envíos: el primero con la nómina del mes de marzo de 2003 y el segundo con la del mes siguiente, con el fin de incrementar el número de respuestas.

Se recibieron en total 53 cuestionarios válidos, lo que supone una tasa de respuesta del $48,1 \%$, existiendo representación de todas las divisiones analizadas y, dentro de cada una de ellas, de sus correspondientes departamentos.

La muestra es relativamente joven, ya que el $50 \%$ de los encuestados no pasa de los 40 años, siendo la edad media de 42,68. Por otro lado, la antigüedad media de los empleados de la muestra es bastante alta (17,93 años), por lo que nos encontramos, en general, con una fuerza de trabajo madura en lo que se refiere a su experiencia profesional. Concretamente, aunque existe un porcentaje amplio de plantilla muy joven, con una antigüedad entre 1 y 5 años, la mayor parte de los empleados se 
incorporaron a la empresa hace más de once años ya que un 17\% lleva trabajando entre 21 y 25 años y otro $17 \%$ entre 26 y 30 , existiendo un $7,5 \%$ con más de 30 años de antigüedad.

En lo que se refiere al nivel de formación, el $43 \%$ de la plantilla tiene estudios de formación profesional o equivalentes, mientras que un $34 \%$ tienen titulación universitaria, ya sea de grado medio $(17 \%)$ o de grado superior (17\%). Además un $13,2 \%$ manifiesta haber realizado cursos complementarios de formación una vez finalizado el bachiller. Adicionalmente, los empleados que llevan más tiempo en la empresa son los que menos nivel de formación tienen, mientras que los titulados universitarios se concentran fundamentalmente en los trabajadores con menos de 11 años de antigüedad.

Por tanto, la muestra está compuesta esencialmente por empleados con una antigüedad media en la empresa bastante elevada y con un nivel medio de estudios de bachiller o formación profesional, cuestión esta última que apunta a que los empleados tienen un gran nivel de experiencia basado en el aprendizaje por la práctica de sus actividades durante un gran periodo de tiempo.

\subsection{Medidas de las variables}

Para medir la creatividad se elaboró una escala multi-ítem a partir de los trabajos de Ettlie y O'Keefe (1982), Cummings y Oldham (1997), Tierney et al. (1999) y Zhou y George (2001), que recogía los dos aspectos fundamentales que reúne el concepto, a saber la novedad y la utilidad. Concretamente, se utilizó una escala tipo Likert de siete puntos en la que 1 significaba total desacuerdo con la afirmación que se realizaba y 7 representaba que el empleado estaba totalmente de acuerdo con la aseveración. La medida reflejaba las autovaloraciones de los empleados sobre su habilidad para ser creativos en su trabajo (Tierney y Farmer 2002). Según esto, una puntuación de 1 significaba que el individuo no se valoraba como creativo, mientras que una puntuación de 7 implicaba que el individuo consideraba que era muy creativo. En total la medida constaba de once ítems, de los cuáles, los seis primeros hacían referencia a la novedad, mientras que los cinco restantes trataban de recoger la utilidad de la idea. Para resumir los datos se ha utilizado la media aritmética de los ítems que conforman la medida, llamando CREATIVIDAD al índice resultante $(\alpha=0,96)$. 
El clima de apoyo a la creatividad se midió con una escala de 36 ítems de siete puntos, desarrollada esencialmente a partir de la Escala Siegel de Apoyo a la Innovación (Siegel y Kaemmerer 1978) y el Work Environment Inventory (WEI) desarrollado por Amabile y Gryskiewicz (1989). En ella, el empleado tenía que valorar su grado de acuerdo con las afirmaciones que se realizaban sobre diversos aspectos relacionados con su entorno laboral, de forma que el 1 significaba que estaba totalmente en desacuerdo, mientras que el 7 suponía un total acuerdo. Una vez recodificadas aquellas sentencias redactadas en sentido inverso, las mayores puntuaciones obtenidas implicaban que el empleado tenía una percepción de que su entorno laboral favorecía o apoyaba la creatividad, y a la inversa. Adicionalmente, se codificó cada ítem de acuerdo con el aspecto que intentaba recoger, tal y como se observa en la Tabla 4.1.

Tabla 4.1: Codificación de los ítems de la escala que mide la relación del clima de apoyo a la creatividad

\begin{tabular}{|c|c|}
\hline Código & Ítem \\
\hline AP1 & Nuestros directivos están deseosos de arriesgarse por una buena idea \\
\hline AP2 & $\begin{array}{l}\text { En esta organización las metas están orientadas hacia el desarrollo de nuevos productos, procesos } \\
\text { y servicios }\end{array}$ \\
\hline AP3 & $\begin{array}{l}\text { Esta organización tiene buenos mecanismos para estimular y desarrollar las ideas creativas, es } \\
\text { decir, para que la gente dedique tiempo y recursos a generar ideas nuevas y originales que sean } \\
\text { útiles y factibles }\end{array}$ \\
\hline AP4 & En esta organización, la alta dirección espera que la gente haga trabajo creativo \\
\hline AP5 & En esta organización, se estimula a la gente para que asuma riesgos \\
\hline AP6 & Esta organización puede ser descrita como flexible y en continua adaptación al cambio \\
\hline AP7 & En esta organización, siempre estamos experimentando nuevas ideas \\
\hline AP8 & $\begin{array}{l}\text { Los miembros de esta organización se dan cuenta de que al tratar con nuevos problemas y tareas, } \\
\text { la frustración es muchas veces inevitable; por tanto esta situación es manejada constructivamente }\end{array}$ \\
\hline EVAL9 & $\begin{array}{l}\text { El trabajo en esta organización es evaluado sobre la base de los resultados, no en función del } \\
\text { esfuerzo realizado }\end{array}$ \\
\hline EVAL10 & En esta organización se hacen muchas críticas a las ideas que se proponen \\
\hline EVAL11 & $\begin{array}{l}\text { La evaluación de las nuevas ideas se basa en la contribución que aportan y no en el lugar que } \\
\text { ocupa el que la sugiere en la jerarquía organizativa }\end{array}$ \\
\hline EVAL12 & $\begin{array}{l}\text { Las nuevas ideas pueden proceder de cualquier parte (departamento, persona) de la organización y } \\
\text { son igualmente bien recibidas }\end{array}$ \\
\hline AP13 & $\begin{array}{l}\text { En esta organización no se castigan las acciones bienintencionadas que terminan en fracaso, sino } \\
\text { que se estimula el aprendizaje por el error }\end{array}$ \\
\hline AP14 & $\begin{array}{l}\text { La habilidad de los miembros de esta empresa para sugerir ideas y métodos originales de hacer las } \\
\text { cosas es respetada y estimada por la alta dirección }\end{array}$ \\
\hline UN15 & $\begin{array}{l}\text { La gente que manda en esta organización, normalmente consigue su buen nombre a cuenta de las } \\
\text { ideas de otros }\end{array}$ \\
\hline AP16 & $\begin{array}{l}\text { Cuando alguien comete un error hay muchísimas excusas, es decir, la gente tiene miedo a } \\
\text { reconocerlo y asumirlo, por temor a las consecuencias }\end{array}$ \\
\hline COMP17 & En general puedo afirmar que me siento identificado con las metas de esta organización \\
\hline COMP 18 & En general, tengo un sentimiento de compromiso con esta organización \\
\hline COMP 19 & En la mayoría de los casos estoy de acuerdo con cómo hacemos las cosas en esta organización \\
\hline
\end{tabular}




\begin{tabular}{ll}
\hline Código & \\
\hline COMP 20 & $\begin{array}{l}\text { Los miembros de esta organización estamos dispuestos a aportar tiempo y esfuerzo extra de } \\
\text { nuestra parte cuando es necesario }\end{array}$ \\
\hline REC21 & La gente es recompensada por el trabajo creativo en esta organización \\
\hline REC22 & La organización reconoce públicamente a los empleados que son creativos \\
\hline FOR23 & $\begin{array}{l}\text { La empresa proporciona formación adecuada de cara a desarrollar el conocimiento y las } \\
\text { habilidades necesarias para generar ideas nuevas, originales y que pueden ser puestas en práctica } \\
\text { (creativas) }\end{array}$ \\
\hline RR24 & Hay recursos adecuados dedicados a la innovación en esta organización \\
\hline RR25 & No hay suficiente financiación para investigar las ideas nuevas y útiles en esta organización \\
\hline RR26 & No cuento con personal suficiente para realizar el proyecto que tengo asignado en la actualidad \\
\hline RR27 & Esta organización me da tiempo libre para perseguir ideas creativas durante el día de trabajo \\
\hline RR28 & Generalmente, puedo conseguir los recursos que necesito para mi trabajo \\
\hline UN29 & \begin{tabular}{l} 
En esta organización hay un flujo activo y vivaz de ideas en todos los niveles \\
\hline UN30
\end{tabular} \begin{tabular}{ll}
$\begin{array}{l}\text { En esta organización, se dispone fácilmente de ayuda y colaboración para el desarrollo de nuevas } \\
\text { ideas }\end{array}$ \\
\hline UN31 & Los distintos grupos/unidades/departamentos de esta organización cooperan entre sí \\
\hline UN32 & $\begin{array}{l}\text { En general, la gente en esta organización tiene una visión compartida de lo que estamos intentando } \\
\text { hacer }\end{array}$ \\
\hline UN33 & En esta organización prevalece una atmósfera amigable entre la gente \\
\hline COMP34 & $\begin{array}{l}\text { En esta organización, los canales de comunicación son abiertos y la información relevante está } \\
\text { fácilmente disponible en todos los niveles }\end{array}$ \\
\hline AP35 & $\begin{array}{l}\text { Ante una situación concreta se permite que los expertos en el tema tomen las decisiones, aunque } \\
\text { esto signifique eludir temporalmente la línea de autoridad formal }\end{array}$ \\
\hline AP36 & $\begin{array}{l}\text { Esta organización suele adaptarse a las circunstancias cambiantes sin que haya demasiado interés } \\
\text { por las prácticas adoptadas en el pasado }\end{array}$ \\
\hline AP = Apoyo a la innovación. EVAL = Evaluación. UN = Unidad y colaboración. COMP = Compromiso. \\
REC = Recompensas y reconocimiento. FOR = Formación. RR = Provisión de recursos.
\end{tabular} \\
\hline
\end{tabular}

Como se ha señalado previamente, el clima es una variable multidimensional que recoge diversos aspectos relativos a la situación organizativa en la que se desenvuelven los empleados. Por este motivo, para reducir los datos, se decidió realizar un análisis factorial por el método de componentes principales, con el fin de obtener las dimensiones o factores que sintetizaran las principales políticas o procedimientos seguidos por la empresa para favorecer la creatividad.

Antes de realizar dicho análisis, se comprobó si los datos disponibles eran adecuados para la aplicación de esta técnica de análisis.

Tal y como puede apreciarse en la Tabla 4.2, tanto el contraste KMO, como la prueba de esfericidad de Barlett arrojaron resultados muy satisfactorios. La MSA resultó también ser adecuada, al obtenerse en la diagonal de la matriz anti-imagen valores superiores a 0,7 . Igualmente, los coeficientes de correlación de esta matriz resultaron todos muy bajos. Por tanto, se confirma la adecuación del análisis factorial como método de reducción y resumen de los datos originales. 
Tabla 4.2: KMO y prueba de Barlett. Clima de apoyo a la creatividad

\begin{tabular}{lrr}
\hline MEDIDA DE ADECUACIÓN MUESTRAL DE KAISER-MEYER-OLKIN &, 863 \\
\hline \multirow{2}{*}{ PRUEBA DE ESFERICIDAD DE BARTLETT } & Chi-cuadrado & 2157,122 \\
& Sig. & 0,000 \\
\hline
\end{tabular}

Una vez comprobado el cumplimiento de los supuestos de adecuación de los datos, se procedió a realizar el ACP. La solución inicial obtenida fue sometida a una rotación ortogonal por el método de la normalización varimax con Kaiser. Al no tener previamente definidas las dimensiones que se querían obtener, se utilizaron los criterios de contraste de caída o cambio de pendiente en el gráfico de sedimentación y el criterio de raíz latente (autovalores superiores a uno) para la extracción de los factores. Ambos criterios aconsejaban la retención de cinco componentes, solución que se aceptó. La matriz de componentes rotados se refleja en la Tabla 4.3, junto con las comunalidades, los autovalores iniciales y el porcentaje de varianza acumulado explicado por cada componente. Como puede observarse, los cinco factores explican casi el 78\% de la varianza total y las comunalidades son muy elevadas, por lo que nos indica la adecuada representación de las variables. Además la matriz presenta un número suficiente de cargas factoriales significativas superiores a 0,5 .

Los factores resultantes del análisis de componentes principales pueden interpretarse como sigue:

- En el caso del primer factor, los ítems más relevantes son aquellos que han sido caracterizados en la literatura como propios de un entorno que apoye las nuevas ideas, en una organización abierta al cambio en constante adaptación al entorno y tolerante con la diversidad de sus miembros. Además incluye el grado en que los empleados perciben que en la organización existe suficiente provisión de recursos (personal, financiación, tiempo) adecuados para la generación de nuevas ideas, junto con una formación adecuada y un flujo continuo de ideas en todos los niveles. Todo esto responde a una orientación empresarial hacia la generación de nuevas ideas que fomenten el desarrollo de nuevos productos, procesos y servicios, con mecanismos y recursos suficientes para estimular y desarrollar dichas ideas, por lo que se ha optado por denominar a este factor ORIENTACIÓN INNOVADORA. 
Tabla 4.3: Clima de apoyo a la creatividad. Matriz de componentes rotados y comunalidades

\begin{tabular}{|c|c|c|c|c|c|c|}
\hline \multirow{2}{*}{ ÍTEMS } & \multicolumn{5}{|c|}{ COMPONENTES } & \multirow{2}{*}{ Сом. } \\
\hline & 1 & 2 & 3 & 4 & 5 & \\
\hline AP1 & 0,804 & 0,225 & 0,218 & 0,284 & 0,209 & 0,868 \\
\hline AP2 & 0,707 & 0,244 & 0,281 & 0,295 & $7,881 \mathrm{E}-02$ & 0,732 \\
\hline AP3 & 0,604 & 0,400 & 0,274 & 0,399 . & $-9,929 \mathrm{E}-03$ & 0,759 \\
\hline AP4 & 0,645 & 0,391 & 9,393E-02 & 0,505 & $3,158 \mathrm{E}-02$ & 0,834 \\
\hline AP5 & 0,821 & 0,230 & 0,313 & $9,428 \mathrm{E}-02$ & 0,131 & 0,851 \\
\hline AP6 & 0,761 & 0,220 & $7,095 \mathrm{E}-02$ & 0,169 & 0,234 & 0,717 \\
\hline AP7 & 0,759 & 0,417 & $3,211 \mathrm{E}-02$ & 0,128 & 0,226 & 0,818 \\
\hline AP8 & 0,722 & 0,118 & 0,309 & 0,251 & 0,295 & 0,781 \\
\hline EVAL9 & 0,235 & 0,205 & 0,175 & 0,203 & 0,792 & 0,797 \\
\hline EVAL10 & 0,431 & 0,399 & 0,209 & $7,178 \mathrm{E}-03$ & 0,590 & 0,630 \\
\hline EVAL11 & $8,699 \mathrm{E}-02$ & 0,131 & 0,752 & 0,475 & 0,162 & 0,842 \\
\hline EVAL12 & 0,319 & 0,312 & 0,705 & 0,322 & 0,108 & 0,811 \\
\hline AP13 & 0,206 & 0,156 & 0,746 & $2,223 \mathrm{E}-02$ & 0,394 & 0,779 \\
\hline AP14 & 0,510 & 0,386 & 0,264 & 0,478 & 0,259 & 0,774 \\
\hline UN15 & 6,791E-02 & 0,661 & 0,110 & 0,265 . & 0,476 & 0,750 \\
\hline AP16 & 0,175 & 0,407 & 0,190 & 0,118 & 0,704 & 0,742 \\
\hline COMP17 & 0,298 & 0,677 & 0,175 & $0,311$. & 0,319 & 0,776 \\
\hline COMP18 & 0,317 & 0,757 & 0,312 & 0,149 & 0,211 & 0,837 \\
\hline COMP19 & 0,453 & 0,602 & 0,314 & 0,122 & $4,785 \mathrm{E}-02$ & 0,683 \\
\hline COMP20 & 0,223 & 0,675 & 0,140 & 0,386 & $6,381 \mathrm{E}-02$ & 0,678 \\
\hline REC21 & 0,422 & 0,320 & 0,294 & 0,643 & 0,262 & 0,849 \\
\hline REC22 & 0,307 & 0,309 & $4,618 \mathrm{E}-02$ & 0,785 & 0,166 & 0,836 \\
\hline FOR23 & 0,557 & 0,336 & 0,300 & 0,403 & 0,139 & 0,695 \\
\hline RR24 & 0,629 & 0,326 & 0,330 & 0,371 . & 0,266 & 0,820 \\
\hline RR25 & 0,614 & 0,223 & 0,320 & 0,423 & 0,366 & 0,841 \\
\hline RR26 & 0,588 & 0,389 & $9,805 \mathrm{E}-02$ & 0,140 & 0,417 & 0,700 \\
\hline RR27 & 0,399 & 0,429 & 0,389 & 0,448 & 0,302 & 0,786 \\
\hline RR28 & 0,521 & 0,421 & 0,242 . & 0,422 . & 0,344 & 0,803 \\
\hline UN29 & 0,552 & 0,463 & 0,229 . & 0,314 . & 0,359 & 0,799 \\
\hline UN30 & 0,617 & 0,493 & 0,327 & 0,259 & 0,336 & 0,911 \\
\hline UN31 & 0,399 & 0,706 & 0,242 & 0,210 & 0,170 & 0,790 \\
\hline UN32 & 0,401 & 0,532 & 0,377 & 0,199 & 0,429 & 0,810 \\
\hline UN33 & 0,300 & 0,679 & 0,122 & 0,208 & 0,365 & 0,743 \\
\hline COMP34 & 0,294 & 0,688 & 0,114 & 0,161 & 0,338 & 0,713 \\
\hline AP35 & 0,410 & 0,224 & 0,636 & $-3,537 \mathrm{E}-02$ & $3,803 \mathrm{E}-02$ & 0,718 \\
\hline AP36 & 0,532 & 0,467 & 0,457 & $-6,797 \mathrm{E}-02$ & $9,581 \mathrm{E}-02$ & 0,793 \\
\hline \% Var.Acu. & 25,737 & 45,386 & 56,906 & 67,731 & 77,957 & \\
\hline Autovalores & 22,216 & 1,969 & 1,524 & 1,281 & 1,075 & \\
\hline
\end{tabular}

- El segundo factor extraído recoge principalmente aquellos ítems relacionados con un ambiente de unidad y colaboración entre los miembros de la empresa, así como con un sentimiento de compromiso 
y de pertenencia a la organización, y de identificación con las metas organizativas. Por este motivo se ha asignado a este factor el nombre de UNIDAD Y COMPROMISO.

- El factor que figura en tercer lugar está explicado fundamentalmente por ítems que hacen referencia a la percepción de igualdad en la evaluación de las ideas con independencia de su procedencia, así como a la posibilidad de que las decisiones las adopten aquellos expertos en un tema eludiendo, si es necesario, la línea de autoridad formal. De ahí que el tercer factor haya recibido el nombre de IGUALDAD.

- El cuarto factor recibe puntuaciones especialmente altas para los dos ítems que reflejan el reconocimiento y las recompensas de la organización a los empleados creativos, por lo que se le dio el nombre de RECONOCIMIENTO.

- Por último, el quinto factor está integrado por tres indicadores que hacen referencia a una evaluación del personal en base al esfuerzo realizado, en la que no se hacen críticas negativas a las ideas propuestas y no existe temor a cometer errores por temor a las consecuencias. Este factor fue denominado EVALUACIÓN DE APOYO.

Una vez finalizado el resumen y la reducción de datos relativos al clima de apoyo a la creatividad, se guardaron las puntuaciones factoriales de cada componente mediante el método de regresión, con el fin de emplearlas en el análisis posterior.

\subsection{Resultados}

Para el contraste de la hipótesis formulada se realizó un análisis de regresión lineal multivariante, utilizando el método paso a paso o stepwise. Los resultados que muestra la Tabla 4.4 permiten comprobar el rechazo de la hipótesis nula de los parámetros, de acuerdo con el test de la F de Ficher Snedecor, para un nivel de significación del 0,01. Por lo tanto, el modelo se ajusta a los datos.

Igualmente, el coeficiente de determinación $\mathrm{R}^{2}$, nos indica que las variables incluidas en el modelo explican en un casi un $53 \%$ el comportamiento de la variable dependiente. No obstante, es evidente que existen más factores de carácter organizativo que influyen en la creatividad, y que no han sido incluidos en el modelo. 
Tabla 4.4: Resumen del modelo: estadístico $F$ y coeficientes de determinación. Clima

\begin{tabular}{ccccc}
\hline Modelo & $\mathbf{R}^{2}$ cuadrado & $\mathbf{R}^{2}$ corregida & $\mathbf{F}$ & Sig. \\
\hline 1 & $0,297^{\mathrm{a}}$ & 0,283 & 21,535 & $0,000^{\mathrm{a}}$ \\
2 & $0,425^{\mathrm{b}}$ & 0,402 & 18,467 & $0,000^{\mathrm{b}}$ \\
3 & $0,480^{\mathrm{c}}$ & 0,448 & 15,068 & $0,000^{\mathrm{c}}$ \\
4 & $0,529^{\mathrm{d}}$ & 0,490 & 13,504 & $0,000^{\mathrm{d}}$ \\
\hline
\end{tabular}

(a) Variables predictoras: (Constante), UNIDAD Y COMPROMISO

(b) Variables predictoras: (Constante), UNIDAD Y COMPROMISO, EVAL. APOYO

(c) Variables predictoras: (Constante), UNIDAD Y COMPROMISO, EVAL. APOYO, IGUALDAD

(d) Variables predictoras: (Constante), UNIDAD Y COMPROM, EVAL APOYO, IGUALDAD, ORIENTACIÓN INNOVADORA

(e) Variable dependiente: CREATIVIDAD

Adicionalmente, se muestran los coeficientes $\mathrm{R}^{2}$ corregidos o ajustados. Como puede observarse, a medida que se incluyen variables en la ecuación, su valor aumenta, creciendo de forma similar que el coeficiente de determinación. Por tanto, queda establecida la relevancia y el poder explicativo de las variables recogidas en la ecuación de regresión final.

En la Tabla 4.5 figuran los coeficientes de regresión asociados a cada una de las variables independientes, junto con los niveles de significación correspondientes. Además se muestran las variables excluidas del modelo con los parámetros y niveles de significación obtenidos.

Tabla 4.5: Coeficientes del análisis de regresión. Clima

\begin{tabular}{|l|c|c|c|c|c|}
\hline & \multicolumn{2}{|c|}{ VARIABLES EXPLICATIVAS } \\
& $\begin{array}{c}\text { Coeficientes no } \\
\text { estandarizados }\end{array}$ & $\begin{array}{c}\text { Coeficientes } \\
\text { estandarizados }\end{array}$ & & \\
\cline { 1 - 4 } & B & Error típ. & Beta & t & Sig. \\
\hline (Constante) & 4,743 & 0,112 & & 42,545 & 0,000 \\
\hline UNIDAD Y COMPROMISO & 0,620 & 0,113 & 0,545 & 5,503 & 0,000 \\
\hline EVAL. DE APOYO & 0,407 & 0,113 & 0,358 & 3,613 & 0,001 \\
\hline IGUALDAD & 0,267 & 0,113 & 0,235 & 2,369 & 0,022 \\
\hline ORIENTACIÓN INNOVADORA & 0,253 & 0,113 & 0,223 & 2,250 & 0,029 \\
\hline
\end{tabular}


A la vista de los resultados, podemos decir que la percepción que tenga el empleado de un ambiente de unidad, colaboración y compromiso, el hecho de que perciba que en la organización se lleva a cabo una evaluación de apoyo y, en menor medida, la apreciación de que sus ideas van a ser bien recibidas con independencia del lugar que ocupe en la escala jerárquica, dejándole incluso tomar decisiones cuando es necesario, junto con la consideración de que la empresa desarrolla prácticas y políticas orientadas a apoyar la creatividad y la innovación en la empresa, tienen un efecto positivo y significativo sobre su nivel de creatividad.

Estos resultados están en consonancia con los obtenidos en la literatura académica, antes señalados, que han analizado cada una de estas variables por separado, así como con aquellos estudios que han utilizado el clima como variable independiente obteniendo posteriormente un número mayor o menor de dimensiones, algunas de ellas coincidentes con las aquí conseguidas.

Sin embargo, no parece que el hecho de que el empleado perciba que la empresa reconozca y recompense a los empleados creativos tenga influencia sobre su creatividad. Este hecho, puede tener su origen en que los empleados creativos revelan elevados niveles de motivación intrínseca, como se ha comprobado anteriormente. Por ello, es factible que la presencia de motivadores extrínsecos no afecte, en principio, a su grado de creatividad, dada la pasión que sienten por el trabajo en sí mismo y que les impulsa a ser creativos. No obstante, esto no quiere decir que se deba dejar de reconocer y recompensar a aquellos empleados que generen ideas novedosas y útiles, puesto que este hecho puede servir de acicate a los trabajadores que, por sus características, no tienen tanta facilidad para hacer nuevas propuestas. Por ello, puede decirse que se confirma parcialmente la hipótesis formulada.

Una vez realizado el análisis de regresión, se procedió a la comprobación del cumplimiento de los supuestos necesarios para la aplicación de la técnica. En las siguientes Tablas (4.6 a 4.8) se muestran los resultados que permiten confirmar la ausencia de multicolinealidad entre las variables explicativas, así como la normalidad e independencia de los residuos. La homocedasticidad de los residuos se comprobó mediante el examen visual de los gráficos de residuos tipificados con respecto a los valores dependientes pronosticados, también tipificados. La anchura de banda de los residuos se mantenía constante cumpliéndose, por tanto, este requisito. 
Tabla 4.6: Multicolinealidad: tolerancia y factor de inflación de la varianza. Clima

\begin{tabular}{lcc}
\hline MODELO & TOLERANCIA & FIV \\
\hline UNIDAD Y COMPROMISO & 1,000 & 1,000 \\
EVAL. DE APOYO & 1,000 & 1,000 \\
IGUALDAD & 1,000 & 1,000 \\
ORIENTACIÓN INNOVADORA & 1,000 & 1,000 \\
& 1,000 & 1,000 \\
\hline Variable dependiente: CREATIVIDAD & &
\end{tabular}

Tabla 4.7: Multicolinealidad: índices de condición. Clima

\begin{tabular}{lcc}
\hline MODELO & DIMENSIÓN & ÍNDICE DE CONDICIÓN \\
\hline UNIDAD Y COMPROMISO & 1 & 1,000 \\
EVAL. DE APOYO & 2 & 1,000 \\
IGUALDAD & 3 & 1,000 \\
ORIENTACIÓN INNOVADORA & 4 & 1,000 \\
& 5 & 1,000 \\
\hline Variable dependiente: CREATIVIDAD & &
\end{tabular}

Tabla 4.8: Independencia y normalidad. Estadístico Durbin-Watson y test de Kolmogorov-Smirnov para los residuos. Clima

\begin{tabular}{ccc} 
Durbin-Watson & Kolmogorov-Smirnov & \\
\multirow{2}{*}{1,669} & $\mathrm{Z}$ & 0,525 \\
& Sig. Asintótica (bilateral) & 0,946
\end{tabular}

Variable dependiente: CREATIVIDAD

\section{CONCLUSIONES}

Los resultados logrados apuntan que efectivamente el clima de apoyo a la creatividad influye positivamente en la creatividad, destacando la importancia de esta variable en la provisión de ideas creativas por parte de los empleados. No obstante el coeficiente de determinación obtenido señala que existen otros elementos del entorno laboral que no han sido incluidos en la medida del clima utilizada.

Por otro lado, el apoyo a la hipótesis formulada es parcial, puesto que la influencia de una de las dimensiones contempladas, concretamente el RECONOCIMIENTO, si bien fue positiva, no resultó significativa. Del resto de los factores, la percepción de unidad y compromiso es el que mayor influencia tiene en la creatividad, seguida por la evaluación de apoyo $\mathrm{y}$, en menor medida, la igualdad y la orientación innovadora de la empresa. 
Por tanto, puede concluirse que la percepción de un ambiente laboral caracterizado por la existencia de unidad y colaboración entre los miembros de la organización, junto con un sentimiento de compromiso y pertenencia a la organización, al tiempo que se siente identificado con las metas organizativas influye positiva y significativamente en la creatividad individual.

Adicionalmente, la percepción de que en la organización se valora el esfuerzo realizado en la consecución de las metas organizativas, incluso aunque los resultados no hayan sido los esperados, de que no se hacen críticas negativas a las ideas que se proponen sino que, por el contrario todas ellas son bien recibidas y de que no hay miedo a cometer errores por temor a las consecuencias influye positiva y significativamente en la creatividad.

Sucede lo mismo con la percepción de que las ideas se valoran y evalúan por igual con independencia de su procedencia y de que existe delegación en la toma de decisiones en expertos eludiendo, si se hace necesario, la línea jerárquica. Esta sensación de igualdad influye positiva y significativamente en la creatividad.

Por último, la percepción de que las prácticas y política implantadas en la organización se caracterizan por fomentar la comunicación fluida entre todos los niveles jerárquicos, proporcionar los recursos materiales necesarios para el desarrollo de nuevas ideas, proveer de la formación adecuada para la adquisición de nuevos conocimientos y de habilidades creativas, además de considerar que la organización está abierta el cambio, en constante adaptación al entorno y es tolerante con la diversidad de sus miembros, influye positiva y significativamente en la creatividad individual.

Sin embargo, no parece que la percepción de que la empresa reconozca y además recompense a los empleados creativos influya en modo alguno en la creatividad de éstos. Este resultado, si bien es contrario a los aportados por la evidencia empírica, puede tener su explicación en el hecho de que los empleados creativos muestran elevados niveles de motivación intrínseca, atributo que en este trabajo ha demostrado ejercer un influjo especialmente relevante en la creatividad. Por este motivo, puede ocurrir que la presencia de motivadores extrínsecos no afecte ni positiva ni negativamente a su creatividad, de forma que el grado de interés y la pasión que sienten por el trabajo que realizan les impulse a utilizar sus habilidades al servicio de la creatividad, con independencia de 
que se les recompense o no por ello. Esto no implica que no haya que articular mecanismos que recompensen o reconozcan el rendimiento creativo, desde el momento que pueden servir de estímulo o motivación a aquellos trabajadores que, bien por carecer de los atributos necesarios, bien por poseerlos pero no estar motivados para ello, no generan habitualmente ideas creativas.

\section{LIMITACIONES}

Sin ninguna duda, esta investigación adolece de limitaciones que deben ser tenidas en cuenta a la hora de interpretar los resultados, con el fin de precisar el alcance de las conclusiones extraídas. A continuación se exponen aquellas que pueden ser más importantes.

Así, la primera limitación a señalar, tal vez por ser la más evidente, es el reducido tamaño de la muestra conseguida. Este hecho, hace que los resultados deban interpretarse desde un principio con prudencia, si bien es cierto que, en general, vienen a apoyar la evidencia obtenida al respecto en la literatura. La consecuencia es que las conclusiones conseguidas únicamente puedan generalizarse al caso de la empresa analizada y, siendo muy optimistas, a empresas de características similares.

Esta limitación está provocada en parte por las características de la empresa en la que se lanzó el cuestionario. En primer lugar se trata de una empresa de fabricación con una plantilla propia ya de por sí reducida. A este hecho hay que añadirle que, desde un principio, fueron excluidos del estudio los trabajadores de la cadena de montaje por para evitar entorpecer el desarrollo de su actividad principal. Esto ha provocado que la población inicial sea pequeña, por lo que, aunque la tasa de respuesta ha sido relativamente alta $(48,1 \%)$, la realidad es que se ha podido contar con muy pocos casos para poder realizar el análisis empírico.

Por otro lado, el propio diseño de la investigación constituye en sí mismo otra importante limitación. En primer lugar, hay que señalar el método elegido para la recogida de información. Si bien el cuestionario era la forma más rápida y barata de obtener la información necesaria para medir las variable del modelo y tener acceso todos los empleados de la empresa incluidos en el estudio, no cabe duda de que su utilización implica aceptar, al tiempo que sus ventajas, los numerosos inconvenientes que plantea, como el no conseguir la implicación de todos los trabajadores 
o lograr información complementaria que podría obtenerse durante la realización de una entrevista personal. No obstante, la decisión de utilizar esta técnica fue impuesta por la empresa para entorpecer lo menos posible el trabajo diario de sus empleados.

Otra limitación tiene que ver con el carácter subjetivo de las medidas empleadas para hacer operativas las variables. Es cierto que su diseño ha sido realizado a partir de escalas cuya fiabilidad y validez ha sido previamente contrastada en la literatura, pero evidentemente su uso sigue conllevando un elevado grado de subjetividad, tanto a la hora de elegir los ítems que finalmente componen la escala, como en las valoraciones realizadas por el que contesta el cuestionario, por lo que hay que tener en cuenta esta cuestión, a pesar de la bondad de los resultados obtenidos en los análisis de fiabilidad y validez realizados. Esta limitación es más acentuada, si cabe, en el caso de la medida de la creatividad, puesto que el hecho de que sean los propios empleados quienes valoran su rendimiento, sin lugar a dudas, conlleva un elevado sesgo y efecto de benevolencia.

\section{FUTURAS LÍNEAS DE INVESTIGACIÓN}

Las limitaciones señaladas con anterioridad dejan abiertos nuevos caminos para la realización de futuras investigaciones que ayuden a mejorar sustancialmente el presente trabajo.

En primer lugar, el modelo contrastado sólo contempla la influencia de los atributos individuales en la creatividad. Por tanto, existe el propósito de ir incorporando en el futuro otras variables de grupo y organizativas que ayuden a explicar mejor el fenómeno estudiado.

Igualmente, se proyecta realizar el estudio en diversos tipos de empresas de mayor tamaño que la utilizada, para realizar una comparación de los resultados entre ellas en función del tipo de actividad que desarrollen y del perfil de empleados existentes en las mismas. Esto permitiría, además, conseguir una mayor generalización de los resultados.

Adicionalmente, puesto que la intención es que este trabajo resulte de utilidad real para las empresas, se pretenden analizar con más profundidad las prácticas de recursos humanos más adecuadas para estimular la creatividad, con la finalidad de poder ofrecer pautas de actuación en este sentido. 
Por otro lado, el presente trabajo se ha centrado en la creatividad individual, sin considerar sus efectos en la creatividad del grupo y los procesos que se dan dentro de los mismos para aprovechar las sinergias del trabajo en equipo. Se aspira, en un futuro, a ampliar el modelo combinando diferentes niveles de análisis, el individual y el de grupo.

\section{BIBLIOGRAFÍA}

ABBEY, A. \& J.W. DICKSON (1983) "R\&D Work Climate and Innovation in Semiconductors", Academy of Management Journal, 26 (2), pp. 362-368.

AMABILE, T.M. (1982) "Social Psychology of Creativity: A Consensual Assessment Technique", Journal of Personality and Social Psychology, 43 (5), pp. 997-1013.

- (1983) The Social Psychology of Creativity. New York: Springer-Verlag.

- (1988) "A Model of Creativity and Innovation in Organizations", Research in Organizational Behavior, 10, pp. 123-167.

- (1996) Creativity in Context. Oxford, USA: Westview Press.

- (1997) "Motivating Creativity in Organizations: On Doing what You Love and Loving what You Do", California Management Review, 40 (1), pp. 39-58.

- \& R. CONTI (1997) "Environmental Determinants of Work Motivation, Creativity and Innovation: The Case of R\&D Downsizing". R. GARUD, P. NAYAR \& Z. SHAPIRA (Eds.) Technological Innovation, Oversights and Foresights. New York: Cambridge University Press, pp. 111-125.

- \& - (1999) "Changes in the Work Environment for Creativity During Downsizing", Academy of Management Journal, 42 (6), pp. 630-640.

,,-- H. COON, J. Lazenby \& M. Herron (1996) "Assessing the Work Environment for Creativity", Academy of Management Journal, 39 (5), pp. 1154-1184.

-, P. GoldFARB \& S.C. BRACKFIELD (1990) "Social Influences on Creativity: Evaluation, Coaction, and Surveillance", Creativity Research Journal, 3, pp. 6-21.

- \& N. GRYSKIEWICZ (1989) "The Creative Environment Scales: The Work Environment Inventory", Creative Research Journal, 2, pp. 231-254. 
-, K.G. Hill, B.A. Hennessey \& E. Tighe (1994) "The Work Preference Inventory: Assessing Intrinsic and Extrinsic Motivational Orientations", Journal of Personality and Social Psychology, 66, pp. 950-967.

-, J.S. Mueller, W.B. Simpson, C.N. Hadley, S.J. Kramer \& L. Fleming (2002) "Time Pressure and Creativity in Organizations: A Longitudinal Field Study", HBS Working Paper, nº 02-073.

ANDREWS, F.M. (1975) "Social and Psychological Factors which Influence the Creative Process". I.A. TAYLOR \& J.W. GeTZELS (Eds.) Perspectives in Creativity. Chicago: Aldine, pp. 117-145.

ANGLE, H.L. (2000) "Psychology and Organizational Innovation". A.H. VAN DEN Ven, H.L. ANGLE \& M.S. POOLE (Eds.) Research on the Management of Innovation. New York: Oxford University Press, pp. 135-170.

BAER, J. (1994) "Divergent Thinking Is Not a General Trait: A Multidomain Training Experiment", Creativity Research Journal, 7 (1), pp. 35-46.

BAILYN, L. (1985) "Autonomy in the Industrial R\&D Lab", Human Resource Management, 24, pp. 129-146.

BRUNER, J.S. (1964) "The Conditions of Creativity". H.E. GRUBER, G. TERRELL \& M. WeRTHEIMER (Eds.) Contemporary Approaches to Creative Thinking, $3^{\mathrm{a}}$ edición. New York: Prentice Hall, pp. 1-30.

CAmpbell, J.P.; M. Dunette, E.E. LaWler III \& K.E. WeIK, Jr. (1970) Managerial Behaviour, Performance, and Effectiveness. New York: McGraw-Hill.

COHEN, W.M. \& D.A. LeVINTHAL (1990) "Absorptive Capacity: A New Perspective on Learning and Innovation", Administrative Science Quarterly, 35, pp. 128-152.

CSIKSZENTMIHALYI, M. (1988) "Society, Culture, and Person: A Systems View of Creativity". R.J. STERNBERG (Ed.) The Nature of Creativity. New York: Cambridge University Press, pp. 325-339.

- (1990) "The Domain of Creativity". M.A. RunCo \& R.S. AlBERT (Eds.) Theories of Creativity. Newpbury Park, CA: Sage, pp. 190-212.

CUmmings, A. \& G.R. OldHAM (1997) "Enhancing Creativity: Managing Work Contexts for the High Potential Employee", California Management Review, 40 (1), pp. 23-38.

CUmmings, L. (1965) "Organizational Climates for Creativity", Academy of Management Journal, 3, pp. 220-222.

DAMANPOUR, F. (1991) "Organizational Innovation: A Meta-Analysis of Effects of Determinants and Moderators", Academy of Management Journal, 34 (3), pp. 55-590. 
DastMalCHIAN, A. (1986) "Environmental Characteristics and Organisational Climate: An Exploratory Study", Journal of Management Studies, 23 (6), pp. 609-627.

DECI, E.L. \& R.M. RYAN (1985) Intrinsic Motivation and Self-Determination in Human Behavior. New York: Plenum.

DelBeCQ, A.L. \& P.K. MILlS (1985) "Managerial Practices that Enhance Innovation", Organizational Dynamics, 14 (1), pp. 24-34.

EkVALL, G. (1987) "The Climate Metaphor in Organization Theory". B.M. BASS \& P.J.D. DRENTH (Eds.) Advances in Organizational Psychology. Beverly Hills CA: Sage Publications, pp. 177-190.

Ellison, R.L.; B. MCDONALD, L. JAMES, D. Fox \& C.W. TAYLOR (1968) An Investigation of Organizational Climate. Greensboro, NC: Richardson Foundation.

,,$---\&$ C.W. TAYLOR (1969) "Relationship of Organizational Characteristics to Measures of Scientific Performance", $77^{\text {th }}$ Annual Conference of A.P.A., 4 (2), pp. 603-604.

ETTLIE, J.E. \& R.D. O'KeEFE (1982) "Innovative Attitudes, Values and Intentions in Organizations", Journal of Management Studies, 19 (2), pp. 163-182.

FINDLAY, C.S. \& C.J. LUMDSEN (1988) "The Creative Mind: Toward an Evolutionary Theory or Discovery and Innovation", Journal of Social Biological Structure, 11, pp. 3-55.

FORD, C.M. (1995) "Creativity Is a Mystery: Clues from the Investigators' Notebooks". C.M. FORD \& D.A. GIOIA (Eds.) Creative Action in Organizations: Ivory Tower Visions and Real World Voices. Newbury Park, CA: Sage Publications, pp. 12-49.

- (1996) "A Theory of Individual Creative Action in Multiple Social Domains", Academy of Management Review, 21 (49), pp. 1112-1142.

- (1999) "Interpretive Style, Motivation, Ability and Context as Predictor of Executives' Performance", Creativity and Innovation Management, 8 (3), pp. 188-196.

FOREHAND, G. \& B. GILMER (1964) "Environmental Variation in Studies of Organizational Behavior", Psychological Bulletin, 22, pp. 361-382.

FRIEDLANDER, F. \& N. MARGULIES (1969) "Multiple Impacts of Organizational Climate and Individual Value System upon Job Satisfaction", Personnel Psychology, 22, pp. 171-183.

GALBRAITH, J.R. (1982) "Designing the Innovating Organization", Organizational Dynamics, 10, Winter, pp. 5-25. 
GrUBer, H.E. (1988) "The Evolving Systems Approach to Creative Work", Creativity Research Journal, 1 (1), pp. 27-51.

GUILFORD, J.P. (1950) "Creativity", American Psychologist, 5, pp. 444-454.

- (1977) Way beyond the IQ: Guide to Improving Intelligence and Creativity. Buffalo, NY: Creative Education Foundation.

- (1984) "Varieties of Divergent Production", Journal of Creative Behavior, 18, pp. 1-10.

ISAKSEN, S.G. (1987) "An Orientation to the Frontiers of Creativity Research". S. ISAKSEN (Ed.) Frontiers of Creativity Research: Beyond the Basics. Buffalo: Bearly Limited, pp. 1-31.

- \& G. KaUfmanN (1990) "Adaptators and Innovators: Different Perspectives of the Psychological Climate for Creativity", Studia Psychologia, 32, pp. 129-140.

JAMES, L.; L. JAMES \& D. ASHE (1990) "The Meaning of Organizations: The Role of Cognition and Values". B. SCHNEIDER (Ed.) Organizational Climate and Culture. San Francisco, CA: Jossey-Bass Inc., pp. 40-84.

- \& S. SelLS (1981) "Psychological Climate: Theoretical Perspectives and Empirical Research". D. MAGNUSSEN (Ed.) Toward a Psychology of Situations: An Interactional Perspective. Hillsdale, NJ: Erlbaum, pp. 275-295.

JAMES, L.R. \& L.A. JAMES (1989) "Integrating Work Environment Perceptions: Explorations into the Measurement of Meaning", Journal of Applied Psychology, 74, pp. 739-751.

- \& A.P. JONES (1974) "Organizational Climate: A Review of Theory and Research", Psychological Bulletin, 81 (12), pp. 1096-1112.

JONES, A. \& L. JAMES (1979) "Psychological Climate: Dimensions and Relationships of Individual and Aggregated Work Environment Perceptions", Organizational Behavior and Human Performance, 23, pp. 210-233.

JoYCE, W.F. \& J. SLoCum (1982) "Climate Discrepancy: Refining the Concepts of Psychological and Organizational Climate", Human Relations, 35 (11), pp. 951-972.

KANTER, R.M. (1983) The Change Masters. New York: Simon \& Schuster.

- (1984) "Innovation: Our Only Hope for Times Ahead?", Sloan Management Review, 25, pp. 51-55.

- (1986) "Creating the Creative Environment", Management Review, 75, pp. 11-12. 
- (1988) "When a Thousand Flowers Bloom: Structural, Collective, and Social Conditions for Innovation in Organizations", Research in Organizational Behavior, 10, pp. 169-211.

KATZ, D. \& R.L. KAHN (1978) The Social Psychology of Organizations, $2^{\text {nd }}$ ed.. New York: Wiley.

KIMBERLY, J.R. (1981) "Managerial Innovation". P.C. NYSTROM \& W.H. STARBUCK (Eds.) Handbook of Organizational Design. New York, Oxford University Press, pp. 84-104.

- \& M.J. EVANSIKo (1981) "Organizational Innovation: The Influence of Individual, Organizational, and Contextual Factors on Hospital Adoption of Technological and Administrative Innovations", Academy of Management Journal, 24 (4), pp. 689-713.

KOESTLER, A. (1964) The Act of Creation. London: Hutchinson.

KoPELMAN, R.E.; A.P. BRIEF \& R.A. GUZZON (1990) "The Role of Climate and Culture in Productivity". B. SCHNEIDER (Ed.) Organizational Climate and Culture. San Francisco, CA: Jossey-Bass Inc., pp. 282-318.

MCKINNON, D.W. (1962) "The Nature and Nurture of Creativity Talent", American Psychologist, 17, pp. 484-495.

- (1970) "The Personality Correlates of Creativity: A Study of American Architects". P.E. VERNON (Ed.) Creativity: Selected Readings. New York: Penguin Books, pp. 289-311.

- (1978) In Search of Human Effectiveness: Identifying and Developing Creativity. Búfalo, NY: Creative Education Foundation.

MARCH, J.G. \& H.A. SIMON (1958) Organizations. New York: Wiley.

MUMFORD, M.D. (2000) "Managing Creative People: Strategies and Tactics for Innovation", Human Resource Management Review, 10 (3), pp. 313-351.

- \& S.B. GUSTAFSON (1988) "Creativity Syndrome: Integration, Application and Innovation", Psychological Bulletin, 103, pp. 27-43.

Oldham, A. \& G.R. Cummings (1996) "Employee Creativity: Personal and Contextual Factors at Work", Academy of Management Journal, 39 (3), pp. 607-634.

PaOliLlo, J.G. \& W.B. Brown (1978) "How Organizational Factors Affect R and D Innovation", Research Management, 21, pp. 12-15.

PAYNe, R.; D. LANE \& M. JABRI (1990) "A Two Dimensional PersonEnvironment Fit Analysis of the Performance, Effort, and Satisfaction of Research Scientists", British Journal of Management, 1, pp. 45-57. 
- \& D.S. PUGH (1976) "Organizational Structure and Climate". M.D. DUNNETE (Ed.) Handbook of Industrial and Organizational Psychology. Chicago: Randall-McNally, pp. 1125-1173.

PeLZ, D.C. (1956) "Some Social Factors Related to Performance in a Research Organization", Administrative Science Quarterly, 1, pp. 310-325.

PRITCHARD, R.D. \& B.W. KARASIK (1973) "The Effects of Organizational Climate on Managerial Job Performance and Job Satisfaction", Organizational Behavior and Human Performance, 9, pp. 123-146.

REICHERS, A.E. \& B. SCHNEIDER (1990) "Climate and Culture: An Evolution of Constructs". B. SCHNEIDER (Ed.) Organizational Climate and Culture. San Francisco, CA: Jossey-Bass Inc., pp. 5-39.

RHODES, M. (1987) "An Analysis of Creativity". S.C. ISAKSEN (Ed.) Frontiers of Creativity Research. Buffalo: Bearly Limited, pp. 216-222.

Rogers, C. (1954) "Toward a Theory of Creativity", Review of General Semantics, 11 (4), pp. 249-260.

SCHNEIDER, B. (1975) "Organizational Climates: An Essay", Personnel Psychology, 28, pp. 447-479.

SCOTT, S.G. \& R.A. BRUCE (1994) "Determinants of Innovative Behaviour: A Path Model of Individual Innovation in the Work Place", Academy of Management Journal, 37 (3), pp. 580-607.

SHALleY, C.E. (1991) "Effects of Productivity Goals, Creativity Goals, and Personal Discretion on Individual Creativity", Journal of Applied Psychology, 76, pp. 179-185.

- (1995) "Effects of Coaction, Expected Evaluation, and Goal Setting on Creativity and Productivity", Academy of Management Journal, 38 (2), pp. 483-503.

-, L.L. GILSON \& T.C. BLUM (2000) "Matching Creativity Requirements and the Work Environment: Effects on Satisfaction and Intentions to Leave", Academy of Management Journal, 43 (2), pp. 215-223.

-, G.R. OldHAM \& J.F. PORAC (1987) "Effects of Goal Difficulty, Goal Setting Method, and Expected Evaluation on Intrinsic Motivation", Academy of Management Journal, 30, pp. 553-563.

- \& J.E. Perry-Smith (2001) "Effects of Social-Psychological Factors on Creative Performance: The Role of Informational and Controlling Expected Evaluation and Modelling Experience", Organizational Behavior and Human Decision Processes, 84 (1), pp. 1-22. 
Siegel, S. \& W. Kaemmerer (1978) "Measuring the Perceived Support for Innovation in Organizations", Journal of Applied Psychology, 63, pp. 553-562.

STAW, B.M. (1990) "An Evolutionary Approach to Creativity and Innovation". M.A. WEST \& J.L. FARR (Eds.) Innovation and Creativity at Work. Chichester, UK: Wiley, pp. 287-306.

STEIN, M.I. (1974) Stimulating Creativity, vol. 1. New York, USA: Academic Press.

TAGGAR, S. (2002) "Individual Creativity and Group Ability to Utilize Individual Creative Resources: A Multi-level Model", Academy of Management Journal, 45 (2), pp. 315-321.

TAYLOR, C.W. (1963) "Variables Related to Creativity and Productivity in Men in Two Research Laboratories". C.W. TALYLOR \& R. BARRON (Eds.) Scientific Creativity: Its Recognition and Development. New York: Wiley, pp. 513-597.

- (1972) "Can Organizations Be Creative Too?". C.W. TAYLOR (Ed.) Climate for Creativity. Elmsford, NY: Pergamon Press, pp. 1-22.

TESLUK, P.E.; J.L. FARR \& S.R. KLEIN (1997) "Influences of Organizational Culture and Climate on Individual Creativity", Journal of Creative Behavior, 31 (1), pp. 27-41.

TIERNEY, P. \& S.M. FARmer (2002) "Creative Self-Efficacy: Potential Antecedents and Relationship to Creative Performance", Academy of Management Journal, 45 (6), pp. 1137-1148.

-, - \& G.B. GRAEN (1999) "An Examination of Leadership and Employee Creativity: The Relevance of Traits and Relationships", Personnel Psychology, 52, pp. 591-620.

TORRANCE, E.P. (1962) "Developing Creative Thinking Through School Experiences". S.J. PARNES \& H.F. HARDING (Eds.) A Source Book for Creative Thinking. New York: Scribner's, pp. 31-47.

- (1967) "Give the 'Devil' His Dues". J.G. GouAN, G.D. Demos \& E.P. TORRANCE (Eds.) Creativity: Its Educational Implications. New York: Wiley.

- (1974) Torrance Test of Creative Thinking: Norms-Technical Manual. Lexington, MA: Ginn.

TURNIPSEED, D. (1994) "The Relationship between the Social Environment of Organizations and the Climate for Innovation and Creativity", Creativity and Innovation Management, 3 (3), pp. 184-195.

Van Den Ven, A.H. (1986) "Central Problems in the Management of Innovation", Management Science, 32, pp. 590-607. 
WALLAS, G. (1926) The Art of Thought. New York: Harcourt Brace Jovanovich. WEISBERG, R.W. (1986) Creativity, Genius and Other Myths. New York: Freeman.

- (1993) Creativity: Beyond the Myth of Genius. New York: Freeman.

- (1999) "Creativity and Knowledge: A Challenge to Theories". R.J. STERnBerg (Ed.) Handbook of Creativity. Cambridge UK: Cambridge University Press, pp. 226-250.

Wertheimer, M. (1945) Productive Thinking. New York: Harper.

WEST, M.A. \& J.L. FARR (1990) "Innovation at Work". M.A. WeST \& J.L. FARR (Eds.) Innovation and Creativity at Work. Chichester, England: Wiley, pp. 1-13.

WoOdman, R.W.; J.E. SAWYER \& R.W. GRIFFIn (1993) "Toward a Theory of Organizational Creativity", Academy of Management Review, 18 (2), pp. 293-321.

ZALTMAN, G.; R. DUnCAN \& J. HOLBECK (1973) Innovations and Organizations. London: Wiley.

ZHOU, J. \& J.M. GEORGE (2001) "When Job Dissatisfaction Leads to Creativity: Encouraging the Expression of Voice", Academy of Management Journal, 44 (4), pp. 682-697. 